\title{
Mucoepidermoid Parotid Gland Tumor Found on Follow-up Radioiodine Scan for Differentiated Papillary Thyroid Cancer
}

\author{
Chinna Naik and Sandip Basu
}

Radiation Medicine Centre, Bhabha Atomic Research Centre, Tata Memorial Hospital Annexe, Mumbai, Maharashtra, India

\begin{abstract}
This report presents a case of focal uptake in the right parotid gland found on a follow-up whole-body radioiodine scan 1 y after successful ablation of differentiated papillary thyroid carcinoma. After the parotid tumor had been excised, it was proven histopathologically to be a low-grade mucoepidermoid tumor. This case illustrates a ${ }^{131}$ I focus that was falsepositive for thyroid carcinoma. The short period between radioiodine treatment and development of this parotid tumor creates doubt about a causal relationship. Induction of salivary gland tumors by radiation has been reported primarily in the setting of external radiotherapy. In the large volume of thyroid carcinoma patients treated with radioiodine across the world, such an occurrence has been rare.
\end{abstract}

Key Words: low-grade mucoepidermoid tumor; parotid; ${ }^{131} \mathrm{I}$; thyroid carcinoma

J Nucl Med Technol 2017; 45:116-118

DOI: 10.2967/jnmt.117.190777

$\mathbf{F}$ alse-positive uptake on whole-body radioiodine scans of patients with differentiated papillary thyroid carcinoma can result from unrelated etiopathologies and should be evaluated thoroughly. Radioiodine ablation of differentiated papillary thyroid carcinoma has few acute or chronic complications. The most common early complication is sialadenitis $(1,2)$. The salivary glands concentrate radioiodine highly selectively, and this iodine is secreted into saliva at a high concentration, as high as 20-100 times that in the serum (3). The epithelium of intralobular ducts is the primary site of iodine transport into saliva $(4,5)$. Periductal capillaries and ductal epithelium help to extract and concentrate the iodine, respectively. Approximately $24 \%$ of administered ${ }^{131}$ I activity is lost in salivary secretions during each cycle of radioiodine ablation (6).

The few salivary gland neoplasms that have been reported after radioiodine ablation include mucoepidermoid carcinoma (7), non-Hodgkin lymphoma (8), and

\footnotetext{
Received Jan. 25, 2017; revision accepted Mar. 14, 2017.

For correspondence or reprints contact: Sandip Basu, Radiation Medicine Centre, Bhabha Atomic Research Centre, Tata Memorial Hospital Annexe, Jerbai Wadia Rd., Parel, Mumbai, Maharashtra 400 012, India.

E-mail: drsanb@yahoo.com

Published online Apr. 13, 2017.

COPYRIGHT (c) 2017 by the Society of Nuclear Medicine and Molecular Imaging.
}

pleomorphic adenoma (9). Although the occurrence of these neoplasms is rare, it is thought to be proportional to the dose of ${ }^{131}$ I administered. To reduce salivary gland damage after radioiodine therapy, use of sour candy (2) or lemon juice (10) to increase salivation is advised in clinical routine. Increased salivation reduces both the transit time of radioiodine through the parotid and other salivary glands and the radioiodine concentration in the salivary glands. Certain cholinergic drugs, such as pilocarpine or cevimeline, are given for $5 \mathrm{~d}(2 \mathrm{~d}$ before, the day of, and $2 \mathrm{~d}$ after radioiodine treatment) to decrease transit time through the salivary glands. Temporary suspension of anticholinergic medication in relevant medical scenarios is also helpful. Intravenous amifostine (an organic thiophosphate) - a recent addition to this list—can reduce the effect of radiation on the salivary glands after radioiodine treatment $(11)$.

\section{CASE REPORT}

A 17-y-old girl initially presented with right-sided swelling of the anterior neck. Routine sonography showed a small hypoechoic lesion in the right lobe of the thyroid measuring $8.7 \times 7.8 \mathrm{~mm}$ and multiple right-sided neck nodules, the largest measuring $3 \times 1.6 \mathrm{~cm}$. Fine-needle aspiration cytology of the neck nodes showed features suggestive of papillary carcinoma of the thyroid, and fine-needle aspiration cytology of the right lobe of the thyroid demonstrated papillary thyroid carcinoma. The patient underwent total thyroidectomy with bilateral level II to $\mathrm{V}$ neck dissection and bilateral central compartment clearance. The final histopathologic diagnosis was differentiated papillary carcinoma of the thyroid (classic type) with regional nodal metastases, after which the patient was referred to our institute for further management. In view of the histopathologic type and a 24-h uptake of $2.7 \%$ at the time of the pretreatment diagnostic scan, the patient was treated with $5,069 \mathrm{MBq}$ of ${ }^{131} \mathrm{I}$ therapy. Posttherapy scanning showed significant uptake in the neck and bilaterally symmetric uptake in the salivary glands. After $6 \mathrm{mo}$, the patient underwent follow-up whole-body radioiodine scanning, which showed uptake in the neck, and there was a marginally elevated thyroglobulin level. In view of the solitary nonpalpable focus and the patient's reluctance to undergo surgical evaluation, she received treatment with 5,291 MBq of ${ }^{131} \mathrm{I}$, and 


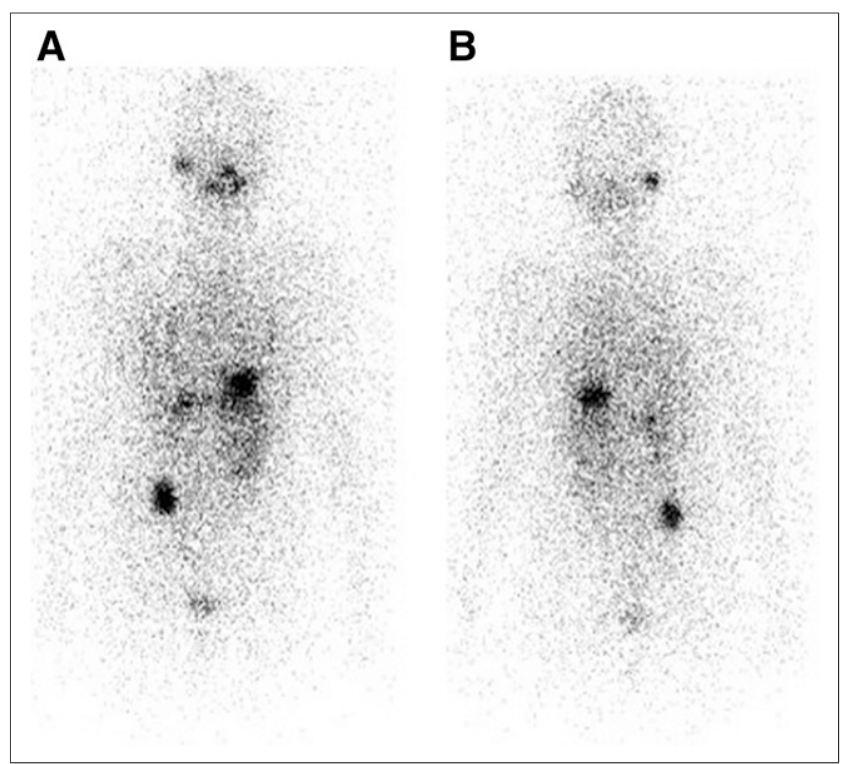

FIGURE 1. Anterior (A) and posterior (B) ${ }^{131}$ I whole-body scans showing asymmetric focal uptake in right parotid gland.

the posttherapy scan showed findings similar to those of the previous scan.

The follow-up whole-body radioiodine scan after $1 \mathrm{y}$ showed successful ablation with no suspected metastatic uptake in the neck, and the level of thyroglobulin was normal. However, there was asymmetric uptake in the salivary gland although no lesion was clinically discernible at that site (Fig. 1). The patient was advised to undergo thyroid-stimulating hormone suppression and to return for follow-up after $1 \mathrm{y}$, according to the routine protocol at our institution. On follow-up, she presented with submandibular swelling that corresponded to the focal uptake on the previous whole-body radioiodine scan. The lesion was excised and found to be a lowgrade mucoepidermoid tumor.

\section{DISCUSSION}

Induction of salivary gland tumors by radiation has primarily been reported in the setting of external radiotherapy. Such tumors may be benign or malignant, with the benign form being more common and comprising mixed tumors or pleomorphic adenomas. The malignant forms that have been reported include mucoepidermoid carcinoma, adenocytic carcinoma, myoepithelial carcinoma, malignant mixed tumor, adenocarcinoma, and acinic cell carcinoma. Modan et al. reported 11 and 21.5 $\mathrm{y}$ as the latency period for the development of malignant and benign salivary gland tumors, respectively (12). In their study, they observed a 4.5-fold increase in incidence of cancer and a 2.6-fold increase of benign tumors. A few studies have reported that the incidence of mucoepidermoid carcinoma increases with the external radiation dose and vice versa: in one review of the literature, the most common histopathologic type for a second malignancy observed in the salivary glands was mucoepidermoid carcinoma (13).

Mucoepidermoid carcinoma of the salivary glands arises from pluripotent reserve cells of the excretory ducts, which can show squamous, columnar, or mucous differentiation (14). Stewart et al. defined the term mucoepidermoid as a tumor with a mixed pattern of two main cell types: epidermoid and mucus-producing (15). Mucoepidermoid tumors are classified as low-, intermediate-, or high-grade. The other characteristics by which mucoepidermoid carcinoma are classified include architectural pattern (cystic vs. solid), cell type (mucous vs. epidermoid), and nuclear pleomorphism. The parotid gland is the most commonly involved among the salivary glands.

Verma et al. (16) reported induction of salivary gland mucoepidermoid carcinoma by external radiation and chemotherapy in 58 patients, most of whom had a tumor of low-grade histopathology localized to the parotid gland. The authors observed a shortened latency period in patients treated with combined chemoradiotherapy versus radiotherapy alone, and the overall 2- and $5-y$ survival rates were $98 \%$ and $93.4 \%$, respectively (16). The main treatment for low-grade salivary gland neoplasms is surgical resection of the primary tumor; neck dissection is the main treatment for the intermediateand high-grade types. Radiotherapy should be recommended for high-grade tumors and tumors with positive margins. Low-grade mucoepidermoid carcinoma, the most common histologic type, has an excellent prognosis after complete resection of the primary tumor.

Our case of mucoepidermoid salivary gland carcinoma after radioiodine treatment is a rare observation. Development of a salivary gland tumor is only one of the possible causes of asymmetric parotid accumulation on posttherapy scintigraphy. However, to err on the side of caution, such suggestive observations should be considered meaningful and included in the report.

\section{CONCLUSION}

We have reported a case of low-grade mucoepidermoid carcinoma found on early follow-up imaging after radioiodine ablation of differentiated thyroid carcinoma. Because of the somewhat short latency period for development of this parotid tumor, a causal relationship with the radioiodine treatment seems doubtful. Asymmetric uptake in the parotid glands on whole-body radioiodine scanning after high-dose radioiodine therapy should be evaluated thoroughly to rule out the possibility of a parotid neoplasm.

\section{DISCLOSURE}

No potential conflict of interest relevant to this article was reported. 


\section{REFERENCES}

1. Allweiss P, Braunstein G, Katz A, Waxman A. Sialadenitis following 1-131 therapy for thyroid carcinoma: concise communication. J Nucl Med. 1984;25: 755-758.

2. Mazzaferri E. Carcinoma of the follicular epithelium. In: Braverman LE, Utiger RD, eds. Werner and Ingbar's The Thyroid. Philadelphia, PA: Lippincott; 2000:904-929.

3. Rigler RG, Scanlon PW. Radiation parotitis from radioactive iodine therapy. Proc Staff Meet Mayo Clin. 1955;30:149-153.

4. Mishkin FS. Radionuclide salivary gland imaging. Semin Nucl Med. 1981;11:258265.

5. Gates GA, Work WP. Radioisotope scanning of the salivary glands. Laryngoscope. 1967;77:861-875.

6. McCall MS, Timm L, Frenkel EP. Chewing tobacco and radioiodine. Lancet. 1967;289:902.

7. Henze M, Hittel JP. Mucoepidermoid carcinoma of the salivary glands after high dosage radiotherapy [in German]. Laryngorhinootologie. 2001;80: 253-256.

8. Wiseman JC, Hales IB, Joasoo A. Two cases of lymphoma of the parotid gland following ablative radioiodine therapy for thyroid carcinoma. Clin Endocrinol (Oxf). 1982;17:85-89.
9. Edmonds CJ, Smith T. The long-term hazards of the treatment of thyroid cancer with radioiodine. Br J Radiol. 1986;59:45-51.

10. Spiegel W, Reiners C, Börner W. Sialadenitis following iodine-131 therapy for thyroid carcinoma. J Nucl Med. 1985;26:816-817.

11. Bohuslavizki KH, Klutmann S, Brenner W, et al. Radioprotection of salivary glands by amifostine in high-dose radioiodine treatment. Strahlenther Onkol. 1999;175(suppl 4):6-12.

12. Modan B, Chetrit A, Alfandary E, et al. Increased risk of salivary gland tumors after low-dose irradiation. Laryngoscope. 1998;108:1095-1097.

13. Beal KP, Singh B, Kraus D, Yahalom J, Portlock C, Wolden SL. Radiationinduced salivary gland tumors: a report of 18 cases and a review of the literature. Cancer J. 2003;9:467-471.

14. Batsakis JG. Salivary gland neoplasia: an outcome of modified morphogenesis and cytodifferentiation. Oral Surg Oral Med Oral Pathol. 1980;49: 229-232.

15. Stewart FW, Foote FW, Becker WF. Muco-epidermoid tumors of salivary glands. Ann Surg. 1945;122:820-844.

16. Verma J, Teh BS, Paulino AC. Characteristics and outcome of radiation and chemotherapy-related mucoepidermoid carcinoma of the salivary glands. Pediatr Blood Cancer. 2011;57:1137-1141.

\section{Erratum}

In "Survey on the Use of Nuclear Renal Imaging in the United States," by Archer and Bolus (J Nucl Med Technol. 2016;44:223-226), the survey was sent to technologists on the National Medicine Technology Certification Board e-mailing list and asked questions about individual departments, with the intent being that all the technologists in a department would coordinate their responses. However, because the responses were anonymous and self-reported, more than one set of responses from the same department might have been received, resulting in more individualexperience data than conglomerate-department data. The authors regret this confounding data collection error.

\section{Publisher's Note}

The page numbering of the March 2017 issue of $J N M T$ was incorrect in the print edition. Please refer to the online edition for the correct page numbers. 